\title{
A Case of Refractory Insomnia Responding to Modified Electroconvulsive Therapy
}

\author{
Xueting Zhang1, Fang Qian², Chengbiao $\mathrm{Lu}^{2,3^{*}}$ \\ ${ }^{1}$ The Outpatient Department of Minkang Hospital of Ningxia Civil Affairs Department, Yinchuan, China \\ ${ }^{2}$ Laboratory of Neuronal Network and Brain Diseases Modulation, School of Medicine, Yangtze University, Jingzhou, China \\ ${ }^{3}$ Key Lab of Brain Research of Henan Province, Department of Physiology and Neurobiology, Xinxiang Medical University, \\ Xinxiang, China \\ Email: *johnlu9000@hotmail.com
}

How to cite this paper: Zhang, X.T., Qian, F. and Lu, C.B. (2017) A Case of Refractory Insomnia Responding to Modified Electroconvulsive Therapy. Yangtze Medicine, 1, 189-193.

https://doi.org/10.4236/ym.2017.14019

Received: July 2, 2017

Accepted: December 10, 2017

Published: December 13, 2017

Copyright (C) 2017 by authors and Scientific Research Publishing Inc. This work is licensed under the Creative Commons Attribution International License (CC BY 4.0).

http://creativecommons.org/licenses/by/4.0/

\begin{abstract}
A 42-year-old female patient suffered refractory insomnia. A variety of drugs including anti-anxiety, antidepressants, antipsychotics and repetitive transcranial magnetic stimulation (rTMS) have been applied in the treatment with no significant effect, whereas modified electroconvulsive therapy (MECT) can significantly improve the patient's sleep.
\end{abstract}

\section{Keywords}

Insomnia, Modified Electroconvulsive Therapy, Major Depression

\section{Introduction}

Based on the Diagnostic and Statistical Manual of Mental Disorders, $4^{\text {th }}$ Edition, Text revision (DSM-IV-TR), the diagnosis of insomnia is defined by a duration of at least 1 month and by symptoms that do not occur exclusively during the course of another sleep disorder, mental disorder, or medical disorder or result from use of substances or medications. The resistant or refractory insomnia can be defined if insomnia treatment with three different treatment options is unsuccessful. The traditional treatment of insomnia includes the use of benzodiazepines and non-benzodiazepine hypnotics [1]. Newly developed compounds such as GABA receptor subtype-selective compounds (S)-3-(aminomethyl)-5methylhexanoic acid (Pregabalin) may overcome the side effects and the risk of drug dependence of classical benzodiazepine [2]. Melatonin, a physiological hormone involved in sleep timing, is currently used exogenously in the treatment of primary and secondary sleep disorders [3] [4].

Beyond drug treatment, clinicians started to explore other effective therapeu- 
tic way including physical stimulation, for example, the modified electroconvulsive therapy (MECT) [5]. MECT is a technically improved electric shock treatment, which is done under general anesthesia and muscle completely relaxed, while monitoring multiple body function parameters including blood pressure, Electroencephalography (EEG), pulse oxygen saturation and respiration.

The MECT has been clinically proven to be a relatively safe physical therapy in psychiatric treatments with fewer complications [6] [7]. Because of its curative effect and relative safety, MECT is widely used in clinical treatments such as schizophrenia (presence of delusions, hallucinations, mania and stupor) and severe depression especially in patients with suicidal tendencies. Recently, it has been reported that MECT has a certain effect in the treatment of severe paradoxical insomnia [5]. However, MECT on long-term refractory insomnia has not been reported. Recently, a long-term insomnia patient was admitted in our hospital and treated with MECT, which showed good response. We here report this case.

\section{Case Report}

Chief Complaint: "More than eight years of poor sleep, worsen in recent three months"

Case history: a 42-year-old female patient came to see doctor at the outpatient department in our hospital in December 2014. Patient claimed her poor sleep appeared in 2008, presented as difficulty in falling asleep, shortened sleep time and light sleep, due to tensive relationship between patient and her husband. She normally slept for 3 - 4 hours at night, sometimes sleeplessness overnight. At daytime, she felt irritated, depressed and headache which seriously affected her performance in the daily work. The patient had a long-term administration of diazepam which was not effective for these symptoms. In 2011, the patient got divorced. Due to the frequent intimidation of her ex-husband, patient claimed that she can only sleep about two hours per night, had fear at night and depressed, accompanied by recurrent skin diseases: seborrheic dermatitis and eczema. In 2012, she went to the doctor at Ningxia mental health Center and was prescribed mirtazapine, clonazepam. After intermittent administration of the two drugs for six months, the patients still suffered poor sleep quality and quitted the medicine due to large side effect. In December 2014, the patient went to our hospital clinic, complaining poor quality of sleep at night, with only two hours of sleep per night.

Physical examination: clear consciousness. Answers to questions were in the point. The mood is low, but no suicidal thoughts.

EEG: brain electrical activity mapping (BEAM) examination and evoked potentials showed no abnormality in mismatch negativity waveform latency, suggesting that in the absence of active attention condition, the brain automatic classification (automatic processing) function to novel stimulation is normal. P300 (P3a, P3b) and N200 (N2) are positive peak waveform that appeared 300 
milliseconds after stimulation and negative waveform 200 - 350 ms after the stimulation respectively, reflecting a variety of cognitive functions such as information processing, reaction, attention and memory. Mild prolonged latency of these waves was observed, suggesting the declined function of control processing, orientation activities and cognition. Contingent negative variation (CNV) demonstrated that the increased arousal level caused attention disorders in the process of expected psychological reaction.

Auditory gating evoked potential P50 (Sensory gating is a normal function of the brain in which the brain inhibits irrelevant sensory stimulation input, so that the advanced function of brain cannot be overloaded by irrelevant stimulation): S2-P50/S1-P50 > 0.5 suggested the brain sensory gating (capacity of resisting disturbance) was missing and the brain cannot rule out irrelevant information.

EEG examination showed increased a waveform in front head. The main component of basic rhythm was the low amplitude $\alpha$ frequency waveform ( 9 - 10 times/sec) combined with $\beta$ frequency waveform (14 - 28 times/sec) and the scattered $\theta$ waveform ( $4-7$ times/sec) with a poor amplitude modulation and no bilateral significant difference.

\section{Drug and TMS Therapy}

Daily oral administration of escitalopram (an antidepressant) $20 \mathrm{mg}$ and Clonazepam (benzodiazepine class of anti-anxiety drugs) $4 \mathrm{mg}$ for two weeks showed poor curative effect and the patient still only sleep 2 - 3 hours per night. Then, drug treatment was combined with transcranial magnetic stimulation (rTMS) at the right frontal lobe dorsolateral area $(1 \mathrm{~Hz}, 80 \%$ of motor threshold), once a day, five times a week, and the treatment last for 12 weeks. The patient undergone this therapy still suffered poor night sleep. Afterwards, tandospirone (anxiolytics), venlafaxine extended release tablets (antidepressants), olanzapine (an antipsychotic) and other drugs were applied with poor curative effect. On November 2015, the patient was recommended to undergo MECT treatment and she agreed to the treatment. Clonazepam administration was stopped right before MECT treatment, but escitalopram was taken $10 \mathrm{mg}$ daily.

\section{MECT Treatment}

The first MECT therapy was initiated on November 23, 2015, the therapy lasted for 6.5 seconds at a power of $35 \mathrm{~J}$ along with drug treatments: atropine $0.5 \mathrm{mg}$, propofol $80 \mathrm{mg}$, succinylcholine chloride $45 \mathrm{mg}$. The moderate convulsion lasted for 116 seconds. The second convulsion lasted for 5 second was observed 10 seconds after first convulsion. The patient was fully awake about 15 minutes after the therapy with good mood and left the treatment room accompanied by a family member.

The patient slept eight hours with good sleep quality at the first night after the therapy. In the following 24 days, she did not undergo MECT treatment and can sleep 4 - 5 hours per night. The $2^{\text {nd }}$ MECT treatment was performed in the $25^{\text {th }}$ 
day, the treatment power was set at $30 \mathrm{~J}$ and power-on time was 5.6 seconds considering the twice convulsion in the first treatment. The convulsive symptom lasted for 120 seconds and EEG showed epileptiform discharges.

The $2^{\text {nd }}$ MECT regimen was performed 3 times per week for 2 weeks. Then, the treatment regimen was changed to once a week for 3 times. During the treatment, the patient slept $4-5$ hours per night with significantly improved mood, alleviated fatigue during the day, normal performance in daily life. After a total of nine treatments, a polysomnography test was performed which showed that the total sleep time was 4 hours 17 minutes, $60.2 \%$ of the total recorded time.

So far, the treatment has been stopped for more than 6 months. The patient claimed no sense of fear, 4 - 5 hours sleep time per night with good mood, normal performance in daily work.

\section{Discussion}

MECT is widely used in the treatment of severe schizophrenia [8] and depression [9] [10]. Less data indicate that MECT can be used in the treatment of insomnia. Insomnia is associated with feelings of restlessness, irritability, anxiety, and daytime fatigue and the symptoms resolve spontaneously after a few days or weeks [11]. However, chronic or refractory insomnia, lasting for three months or longer can reduce individual's function both socially and professionally. Insomnia is believed to be precursor and concurrent symptoms [12] of clinical depression [13]. The treatment of insomnia is an important step in complete alleviation of depression [14].

MECT has achieved good effect in the treatment of severe paradoxical insomnia and depression. However, the patient with chronic insomnia in this case did not have typical symptoms of depression and she mainly suffered chronic insomnia accompanied by the symptoms of fear and slight anxiety. Although the patient has applied drug therapy or drug combined with TMS therapy, it is clear that these treatments had no significant effect on this intractable insomnia. Here, MECT therapy significantly improved sleep quality in a long-term intractable insomnia patient (from $2 \mathrm{hrs}$ to $4-5 \mathrm{hrs}$ ), thus ensuring the normal life of the patient with the ability to work. This has offered new important and effective clinical treatment for the patient with refractory insomnia. As a generally accepted therapy in wide field of psychiatric treatment, MECT is relatively safe and has a rapid action. To our knowledge, this is the first report to describe MECT application in a patient with chronic insomnia.

\section{References}

[1] Rudolph, U. and Knoflach, F. (2011) Beyond Classical Benzodiazepines: Novel Therapeutic Potential of GABAA Receptor Subtypes. Nature Reviews Drug Discovery, 10, 685-697. https://doi.org/10.1038/nrd3502

[2] Di Iorio, G., et al. (2013) Treatment-Resistant Insomnia Treated with Pregabalin. European Review for Medical and Pharmacological Sciences, 17, 1552-1554. 
[3] Benloucif, S., et al. (2008) Measuring Melatonin in Humans. Journal of Clinical Sleep Medicine, 4, 66-69.

[4] Auld, F., et al. (2017) Evidence for the Efficacy of Melatonin in the Treatment of Primary Adult Sleep Disorders. Sleep Medicine Reviews, 34, 10-22. https://doi.org/10.1016/j.smrv.2016.06.005

[5] Case, K., et al. (2008) A Case of Extreme Paradoxical Insomnia Responding Selectively to Electroconvulsive Therapy. Journal of Clinical Sleep Medicine, 4, 62-63.

[6] Chanpattana, W. and Kramer, B.A. (2003) Acute and Maintenance ECT with Flupenthixol in Refractory Schizophrenia: Sustained Improvements in Psychopathology, Quality of Life, and Social Outcomes. Schizophrenia Research, 63, 189-193. https://doi.org/10.1016/S0920-9964(02)00330-4

[7] Grover, S., Hazari, N. and Kate, N. (2015) Combined Use of Clozapine and ECT: A Review. Acta Neuropsychiatrica, 27, 131-142. https://doi.org/10.1017/neu.2015.8

[8] Jiang, Y., et al. (2015) Effects of Modified Electroconvulsive Therapy on the Cognitive Function and Blood Parameters in Female Patients with Schizophrenia. International Journal of Clinical and Experimental Medicine, 8, 1349-1355.

[9] Kucia, K.A., Stepanczak, R. and Tredzbor, B. (2009) Electroconvulsive Therapy for Major Depression in an Elderly Person with Epilepsy. The World Journal of Biological Psychiatry, 10, 78-80. https://doi.org/10.1080/15622970701320582

[10] Jin, X.L., et al. (2016) Long-Term Effectiveness of Modified Electroconvulsive Therapy Compared with Repetitive Transcranial Magnetic Stimulation for the Treatment of Recurrent Major Depressive Disorder. The Journal of Nervous and Mental Disease, 204, 479-482. https://doi.org/10.1097/NMD.0000000000000493

[11] Fiorentino, L. and Martin, J.L. (2010) Awake at 4 AM: Treatment of Insomnia with Early Morning Awakenings among Older Adults. Journal of Clinical Psychology, 66, 1161-1174. https://doi.org/10.1002/jclp.20734

[12] Ford, D.E. and Kamerow, D.B. (1989) Epidemiologic Study of Sleep Disturbances and Psychiatric Disorders. An Opportunity for Prevention? JAMA, 262, 1479-1484. https://doi.org/10.1001/jama.1989.03430110069030

[13] Breslau, N., et al. (1996) Sleep Disturbance and Psychiatric Disorders: A Longitudinal Epidemiological Study of Young Adults. Biological Psychiatry, 39, 411-418. https://doi.org/10.1016/0006-3223(95)00188-3

[14] Fava, M. (2004) Daytime Sleepiness and Insomnia as Correlates of Depression. Journal of Clinical Psychiatry, 65, 27-32. 